\title{
BMJ Open Integrating tobacco cessation into routine dental practice: protocol for a qualitative study
}

\author{
Rachana Shah, ${ }^{1,2}$ Rupal Shah, ${ }^{1}$ Sujal Shah, ${ }^{3}$ Upendra Bhojani ${ }^{4,5}$
}

To cite: Shah $\mathrm{R}$, Shah $\mathrm{R}$, Shah S, et al. Integrating tobacco cessation into routine dental practice: protocol for a qualitative study. BMJ Open 2019;9:e028792. doi:10.1136/ bmjopen-2018-028792

- Prepublication history and additional material for this paper are available online. To view these files, please visit the journal online (http://dx.doi org/10.1136/bmjopen-2018028792).

Received 31 December 2018 Revised 06 July 2019 Accepted 09 July 2019

Check for updates

(c) Author(s) (or their employer(s)) 2019. Re-use permitted under CC BY-NC. No commercial re-use. See rights and permissions. Published by BMJ.

${ }^{1}$ Department of Prosthodontics, Government Dental College and Hospital, Ahmedabad, Gujarat, India

${ }^{2} \mathrm{PhD}$ Student, Gujarat University, Ahmedabad, India

${ }^{3}$ The Smile Makers Dental Clinic, Ahmedabad, India

${ }^{4}$ Faculty and Wellcome Trust/ DBT India Alliance, Institute of

Public Health, Bengaluru, India

${ }^{5}$ Department of Anthropology,

Durham University, Durham, UK

Correspondence to

Dr Rachana Shah;

doc_rach2000@yahoo.com

\section{ABSTRACT}

Introduction Combined efforts to encompass different aspects of tobacco control have been in place for some time. Despite the recognition of the need to offer support to tobacco users to quit tobacco use, such support remains highly inadequate in India. However, little is known about the practice of oral health professionals (OHP) and the experiences and expectations of dental patients in the context of tobacco cessation (TC) services. In this article, we describe the protocol of a doctoral research project that explores OHPs and their patients in an Indian city. The aims are $(A)$ to understand the functioning of the oral healthcare system towards TC and what changes to it will be needed to benefit TC and (B) to capture the views of dental patients on TC services provided by OHPS.

Methods and analysis A cross-sectional qualitative study based on individual interviews with OHPs and dental patients will be carried out in the city of Ahmedabad, Gujarat, India. The OHP will be purposively selected from two major organisation types: (1) single-doctor dental clinics and (2) dental hospital attached to teaching institutions. The sample population will be divided into two subgroups: general OHP (dentists practising general dentistry irrespective of their qualification) and prosthodontists (dentists with a specialisation in prosthodontics). We will sample dental patients through convenient sampling from a public teaching hospital and select private dental care facilities. The sampling of OHPs and dental patients will continue until we reach data saturation. Interviews will be audio recorded, transcribed verbatim and coded by hand. The interview transcript will subsequently be analysed using thematic content analysis. Ethics and dissemination The study received ethical approval from the Institutional Ethical Committee of the Government Dental College and Hospital, Ahmedabad. The findings will be disseminated through conference presentations, peer-reviewed publications and to the study participants.

\section{INTRODUCTION}

India is the second largest consumer of tobacco, with $28.6 \%$ of adults being current users of one or more tobacco products. ${ }^{1}$ In 2010 , tobacco use was estimated to have caused about 1.3 million adult deaths in India. ${ }^{23}$ Tobacco use is associated with several morbidities, including many oral diseases. India has one of the highest incidences of

\section{Strengths and limitations of this study}

The study will allow an in-depth understanding of the challenges and possibilities of tobacco cessation (TC) in the current dental practice of oral health professional (OHP) working in different clinical set-ups.

- The study will generate knowledge that could inform practices of oral healthcare to bring about regulatory or institutional changes and will help in policy development and design resulting in a tailored TC policy for OHPs working in different types of set-ups. It will be useful in informing similar inquiries in other settings and pave the way for future research.

- The study site will limit the relevance of the findings to similar metropolitan areas as smaller towns are likely to present different organisational set-ups and challenges.

- A convenient sampling of patients may not be representative of the patient community in the city at large.

- Self-reported data could vary from the participant's actual behaviour.

oral cancers in the world. ${ }^{4}$ About $90 \%$ of these cancers are related to tobacco use. ${ }^{5}$ Several tobacco control measures taken by the Indian government, like national legislation (Cigarette and Other Tobacco Products Act, 2003), a national programme on tobacco control and ratification of the WHO Framework Convention on Tobacco Control, have pushed up the demand for tobacco cessation (TC) services. The recent nationwide survey suggests that $55.4 \%$ of current smokers and $49.6 \%$ of current smokeless tobacco users are planning to quit tobacco use. ${ }^{1}$ The need to provide TC as a part of primary healthcare is long acknowledged. Research has shown that brief tobacco intervention by oral health professional (OHP) has an impact on the quitting rates of tobacco users. Despite recognition of the need to offer support to stop tobacco use as an important component of the tobacco control policy prescription (MPOWER) by the WHO, such support remains highly inadequate in India. The 
Ministry of Health and Family Welfare (Government of India) with the support of the WHO has started 19 TC clinics in India. ${ }^{6}$ However, the majority of these centres are located in tertiary/superspecialty hospitals. Hence, these centres often end up serving people who have already suffered from severe tobacco use-related harm with very little scope of preventive and early interventions. Through the national tobacco control programme, the government has attempted to organise counselling support in select districts across India. More recently, the national government has started to provide phone-based and mobile phone app-based support to tobacco users.

While these initiatives are very much needed, we are yet to fully optimise the potential that the formal healthcare services offer in terms of providing integrated TC services. At present, only half of current smokers and only a third of current smokeless tobacco users get some advice from healthcare providers for TC. ${ }^{7}$ Hence, there is room for the integration of brief TC intervention in general healthcare services, with oral healthcare services possessing a unique potential. Oral healthcare providers have easy access to tobacco users in the early stages; thus, providing an opportunity to intervene. They often have more chairside time with patients than other clinicians. Moreover, tobacco use prevention also enhances dental treatment outcomes. Some dental specialists, such as prosthodontists, require spending even more time with patients and the need for several follow-ups providing them with an opportunity to reinforce messages on cessation.

While OHPs are known to be very much willing to offer TC advice, they rarely put TC in practice. The major barriers perceived for this gap between their willingness and practice are a lack of training, a lack of time, a lack of financial incentives and, intriguingly, the fear that the patient might get annoyed and consequently stop seeking care from that dental office/facility. ${ }^{8-12}$ There is a lack of research on how to overcome these barriers and integrate cessation into routine dental care. In this paper, we present a protocol for doctoral research that aims to better understand how TC could be integrated into routine dental practice by general OHP and prosthodontists working in single-doctor dental clinics and dental hospitals attached to teaching institutions in Ahmedabad, India.

In order to better understand how to integrate TC into routine dental practice, we aim to address the following specific research questions:

- How is the current TC service (if at all) organised in oral healthcare facilities in Ahmedabad city across major organisation types (single-doctor dental clinics and dental hospitals attached to teaching institutions)?

- What are the suggestions by OHPs with regard to the feasibility of providing integrated TC support within routine dental practice?

- What are the perceived needs and expectations of dental patients with regard to TC support?
- What sort of intervention protocols/prototypes can be designed from this research for TC in routine dental practice?

\section{METHOD}

\section{Study design and setting}

We propose to conduct a cross-sectional qualitative study based on individual semistructured interviews with OHPs and dental patients. The interview topics for the OHP are guided by the WHO health system building blocks, ${ }^{8}$ as well as considering the known barriers for TC in dental practice reported in the literature ${ }^{8-12}$ (online supplementary files 1 and 2). The study will be carried out in Ahmedabad, the largest city in the western Indian state of Gujarat with a population of over 7 million. It is the sixth largest city and the seventh largest metropolitan area in India. The Ahmedabad urban populationbased cancer registry reports the highest proportion of tobacco-related cancer for both males $(56.3 \%)$ and females $(19.8 \%) .{ }^{13}$ The incidence rate of oral cancer among men from the urban population of Ahmedabad has increased markedly from 1985 to $2010 .{ }^{14}$ The oral healthcare services available here are in the form of the government and private care set-up. With more than 1500 private oral healthcare set-ups, the city has two government and three private dental hospitals attached to teaching institutes.

\section{Study sampling}

Aligned with the qualitative nature of the study, a nonprobability sampling approach will be adopted, and no sample size calculation will be done. As representativeness of the findings for an OHP and a dental patient spectrum that is as broad as possible is desired, we will use a variation sampling approach as a variant of purposive sampling. ${ }^{15}$

\section{Oral health professional}

For the sampling of OHPs, we will strive to attain respondents across gender, specialty and the organisational setups that OHPs work in. This OHP spectrum composition will be purposively selected from the two major organisation types that most OHPs work in: (1) single-doctor dental clinics and (2) dental hospitals attached to teaching institutions. The sample population will be divided into two subgroups: general OHP (dentists practising general dentistry irrespective of their qualification) and prosthodontists (dentists with a specialisation in prosthodontics). General OHP and prosthodontists from these two organisational set-ups will be conveniently sampled through the researcher's network and snowballing. The sampling will continue until we reach data saturation, the point when new data (interviews) will repeat information already expressed in the previous data. ${ }^{16}{ }^{17}$ We shall continue interviewing until we complete two interviews where data saturation is evident. The broad inclusion criteria for OHP would be that they have a formally recognised 
qualification and registration with the Dental Council of India and are presently practising dentistry in Ahmedabad city.

\section{Dental patients}

For the dental patients, we will cover age, gender and socioeconomic background characteristics to obtain maximum variation within the spectrum. The patients will be sampled purposively from a public teaching hospital (Government Dental College and Hospital, Ahmedabad, where the first author is affiliated) and select private dental care facilities within the social networks of the authors in Ahmedabad city, representing the two main organisation types. The patient spectrum composition will be selected conveniently from these two organisations. The sampling will continue until we reach data saturation, the point when new data (interviews) will repeat information already expressed in the previous data. ${ }^{16}{ }^{17}$ We shall continue interviewing until we complete two interviews where data saturation is evident. The broad inclusion criteria for patients would be that they are adults (aged 20 years old or above) who are tobacco users and currently undergoing dental treatment or have undergone some dental treatment in the last year. We will exclude patients with debilitating physical and mental conditions. The patient of the teaching institution will be selected and invited for the interview. As the researcher is herself a dentist working in this institute, patients receiving care from her will not be included in the study. The ones from the single-doctor dental clinics will be invited after permission from the OHP working in that clinic.

\section{Data collection}

We will interview sampled OHPs and patients using semistructured interview guides. Both the guides will be field tested by interviewing a small sample of OHPs and patients. It will be further refined for clarity and relevance. The first author will contact (by phone) the sampled OHPs explaining the purpose of the study and securing their appointments. After explaining the study purpose and the voluntary and anonymous nature of participation, the first author will seek their written informed consent. The interviews, with duration of about $30-45 \mathrm{~min}$, will be audio recorded and conducted at a place (that assures privacy and comfort) and time convenient to them. Similarly, after explaining and seeking informed written or thumbprint consent, patients will be interviewed in a room (ensuring privacy and comfort) within the healthcare facility of a teaching institute and in a room provided by OHP of the single-doctor dental clinic after verbal consent. The interviews, with duration of about $20-30 \mathrm{~min}$, will be audio recorded following permission from the participants. The first author, accompanied by a colleague, will conduct all the interviews in any/mix of languages that the participants are most comfortable with.
Interviews will then be transcribed verbatim in the original language and subsequently translated into English. The data collection and analysis, including the report writing, are expected to be done from June 2019 to May 2021.

\section{Ethics and dissemination}

As the researcher is a part of the institute from where the participants are going to be recruited, her position is bound to influence the data coproduced through interviews. The identity of the researcher as an OHP may bring in power dynamics, especially while interviewing patients. While it is not possible to fully eliminate such an influence, the researcher will ensure not to interview any patients with whom the researcher is associated as a caregiver and assure all the respondents that their responses will in no way impact their care and relationship with their OHP. Similarly, there could be dynamics while interviewing participants of opposite gender from that of the interviewer's. This is more likely to be of consequence in case of the interviewed patients compared with the interviewed OHPs. We will also be vigilant of these dynamics while analysing data to identify any clear pattern that can be attributed to such power dynamics.

It is likely that the respondent might be semiliterate or illiterate, in which case, the researcher will read out and explain the consent form. If the respondent agrees to provide consent, their thumbprint will be taken on the consent form. Participants will remain anonymous; their names will be replaced by an ID number during the transcription and their identity will not be disclosed for the report and any subsequent publication. Secure electronic and paper-based filing systems for both the recordings and transcripts will be set up. All the study-related data will be kept for a minimum of 5 years after the end of the study. The findings of the study will be shared with all the stakeholders of this research and will be disseminated through conference presentations and peer-reviewed publications.

\section{Data analysis}

Thematic content analysis will be carried out following the six-phase iterative process suggested by Braun and Clarke: (1) familiarising oneself with data, (2) generating initial codes, (3) searching for themes, (4) reviewing themes, (5) defining and naming themes and (6) providing the report, which will be used to analyse the qualitative data. ${ }^{18-20}$ We will carry out content coding using a mix of the inductive and deductive approaches, that is, while we will look for coding material relevant to WHO health system building blocks themes, we will also openly code material that responds to our research questions. The data will be hand-coded by the main researcher to bring out the themes which will then be discussed with other researchers. The data will be presented as dominant themes explaining research objectives and will explore new ideas to inform future research. 


\section{Patient and public involvement}

Our research question concerning integrating TC in routine dental care is, in part, based on a relative lack of proactive assistance offered by healthcare providers for TC as reported by tobacco users and the general public in a large-scale survey in India. Patients were not involved in the study design, nor will they be involved in the recruitment and conduct of the research. They will be interviewed to better understand their perceived expectations and experiences with regard to TC by their dental care providers. A simple brief summary of the study results and recommendations will be produced and disseminated to concerned stakeholders, including the study participants who opt for receiving one at the time of participation in the study.

\section{DISCUSSION}

Dentistry, in current times, is oriented more towards curative rather than preventive aspects. Studies show that OHPs value the preventive part of dentistry but find it difficult to practice and personally disreputable. ${ }^{21-23}$ Available evidence suggests that brief interventions increase tobacco abstinence rates. ${ }^{24}$ With OHPs being aware of the benefits and the need for TC, the bridge between the willingness and practice is missing. This study will delve into the current practice and organisational set-ups of OHPs to understand the possibilities and potential of integrating TC into routine care. The opinions of dental patients regarding TC will further shape the integration policies for the practice of dentistry. Generating such data will help make policy, regulatory or institutional changes that will be easily acceptable and make the preventive aspect of dentistry more effective.

\section{Strengths and limitations}

The selected study site would limit the relevance of the findings to similar metropolitan areas in the state as smaller towns and rural areas are likely to present different organisational set-ups and challenges with regard to TC practice. A convenient sampling of patients in our study may not be representative of the patient community in the city at large. Self-reported data could vary from the participant's actual behaviour. A single coder approach that we propose to use may introduce bias in coding data compared with independent coding by two or more researchers. However, the qualitative nature of the study allows for an in-depth understanding of the challenges and possibilities of TC in dental practice. Beyond the specific context of the study setting, such work is useful in informing similar inquiries in other settings but is not amenable to the strict generalisation possible in quantitative experiments.

\section{CONCLUSION}

The study explores OHPs, the system in which they work and their patients. Hence, in conclusion, the study will lead to the design of specific protocols for improving the integration of $\mathrm{TC}$ in routine practice.

Correction notice This article has been corrected since it was published. Affiliation added for Dr Rachana Shah.

Contributors RaS and UB conceived the research idea and designed the study. RaS wrote the initial draft of this paper. RuS, SS and UB edited and revised the paper.

Funding UB was supported for his time spent on this paper through the Wellcome Trust /DBT India Alliance Fellowship [IA/CPHI/17/1/503346] awarded to him.

Competing interests None declared.

Patient consent for publication Not required.

Ethics approval This study has been approved by the Institutional Ethical Committee at the Government Dental College and Hospital, Ahmedabad.

Provenance and peer review Not commissioned; externally peer reviewed.

Open access This is an open access article distributed in accordance with the Creative Commons Attribution Non Commercial (CC BY-NC 4.0) license, which permits others to distribute, remix, adapt, build upon this work non-commercially, and license their derivative works on different terms, provided the original work is properly cited, appropriate credit is given, any changes made indicated, and the use is non-commercial. See: http://creativecommons.org/licenses/by-nc/4.0/.

\section{REFERENCES}

1. Ministry of Health and Family Welfare, Government of India. GATS-2 Highlights-Global adult tobacco survey fact sheet India 2016-2017. Available: https://mohfw.gov.in/newshighlights/global-adult-tobaccosurvey-2-gats-2-india-2016-17-report [Accessed 3 Oct 2018].

2. Jha $P$, Jacob B, Gajalakshmi V, et al. A nationally representative case-control study of smoking and death in India. N Engl J Med Overseas Ed 2008;358:1137-47.

3. Sinha DN, Palipudi KM, Gupta PC, et al. Smokeless tobacco use: a meta-analysis of risk and attributable mortality estimates for India. Indian J Cancer 2014;51:73-7.

4. Ganesh R, John J, Saravanan S. Socio demographic profile of oral cancer patients residing in Tamil Nadu - a hospital based study. Indian J Cancer 2013;50:9-13.

5. Control of oral cancer in developing countries. A who meeting. Bull World Health Organ 1984;62:817-30.

6. Effective Implementation of the WHO Framework Convention on Tobacco Control through the MPOWER Policy Package. TFI Newsletter (who SEARO) 2009;2:1-6.

7. Ministry of Health and Family Welfare, Government of India. GATS Highlights-Global adult tobacco survey fact sheet India 2009-2010. Available: http://www.who.int/tobacco/surveillance/india fact sheet. pdf?ua=1 [Accessed 3 Oct 2018].

8. World Health Organization. Who monograph on tobacco cessation and oral health integration. Geneva: World Health Organization, 2017.

9. Shah R, Shah R, Bhojani U, et al. Dentists and tobacco cessation: moving beyond the willingness. J Indian Assoc Public Health Dent 2017;15:263-4.

10. Ahmed Z, Preshaw PM, Bauld L, et al. Dental professionals' opinions and knowledge of smoking cessation and electronic cigarettes: a cross-sectional survey in the North of England. Br Dent J 2018;225:947-52.

11. Lala R, Csikar J, Douglas G, et al. Factors that influence delivery of tobacco cessation support in general dental practice: a narrative review. J Public Health Dent 2017;77:47-53.

12. Watt RG, McGlone P, Dykes J, et al. Barriers limiting dentists' active involvement in smoking cessation. Oral Health Prev Dent 2004;2:95-102.

13. Three year report of the population based cancer registries 2012-2014: report of 27 PBCRs in India. National Cancer Registry Programme, Banglore: Indian Council Medical Research 2016.

14. Gupta PC, Ray CS, Murti PR, et al. Rising incidence of oral cancer in Ahmedabad City. Indian J Cancer 2014;51:67-72.

15. Bowling A. Research methods in health: investigating health and health services. McGraw-Hill International, 2009.

16. O’Reilly M, Parker N. 'Unsatisfactory Saturation': a critical exploration of the notion of saturated sample sizes in qualitative research. Qualitative Research 2013;13:190-7.

17. Walker JL. The use of saturation in qualitative research. Can J Cardiovasc Nurs 2012;22:37-46. 
18. Patton MQ. Qualitative evaluation and research methods. 3rd edn. Thousand Oaks, CA, US: Sage Publisher, 2002.

19. Polit DF, Beck CT. Nursing research: generating and assessing evidence for nursing practice. 9th edn. Philadelphia, PA, US: Wolters Kluwer-Lippincott, Williams \& Wilkins, 2012.

20. Braun V, Clarke V. Using thematic analysis in psychology. Qual Res Psychol 2006;3:77-101.

21. Ahuja N, Krishnamurthy A, Pramila M, et al. Knowledge and attitude towards preventive dental care among dental faculties in Bangalore City. J Indian Assoc Public Health Dent 2014;12:93-9.
22. Ghasemi $\mathrm{H}$, Murtomaa $\mathrm{H}$, Torabzadeh $\mathrm{H}$, et al. Knowledge of and attitude towards preventive dental care among Iranian dentists. Eur $J$ Dent 2007;1:222-9.

23. Khami MR, Murtomaa $\mathrm{H}$, Jafarian $\mathrm{M}$, et al. Knowledge and attitude of Iranian dental school educators towards prevention. Oral Health Prev Dent 2007:5:181-6.

24. Carr AB, Ebbert J. Interventions for tobacco cessation in the dental setting. Cochrane Database Syst Rev 2012;21. 